\title{
Breakdown in short rod-plane air gaps under positive lightning impulse stress
}

\author{
Hans Kristian Hygen Meyer, Frank Mauseth, Martine Husøy \\ Norwegian University of Science and Technology
}

\author{
Jonas Ekeberg \\ $A B B$ Switzerland Ltd.
}

\author{
Atle Pedersen \\ SINTEF Energy Research
}

\begin{abstract}
Prediction of withstand voltages in air-insulated systems are made on the basis of empirical models that are not sufficiently accurate for complex geometries. Better understanding of the spatiotemporal development of electrical discharges is necessary to improve the present models. Discharges in lightning impulse stressed 20$100 \mathrm{~mm}$ rod-plane gaps are examined using a highspeed camera, photo-multiplier tubes (PMTs) and a highbandwidth current measurement system. The images and measurements of gaps larger than $20 \mathrm{~mm}$ show a fast initial streamer discharge with a current rise time of some tens of ns, followed by a dark period of a few $\mu \mathrm{s}$ and a propagation of a slower leader-type channel leading to breakdown. The breakdown mechanisms in the shortest gaps are faster and geometry dependent, probably occuring by heating of initial streamer channels. Different light filters used with the PMTs indicate that all parts of the leader-type discharge development emit light over a spectrum from UV to IR. The initial discharges emit low amounts of warm light and IR compared to the leader-type channel. Finally, it is suggested that empirical breakdown voltage prediction models should be interpreted in light of the leader-type breakdown mechanism.
\end{abstract}

\section{Introduction}

$\mathrm{SF}_{6}$, a very strong greenhouse gas, is often used as insulation in medium voltage (MV) equipment, although insulating gases with lower global warming potential have recently been proposed [1], [2]. Air in combination with dielectrics is a feasible alternative to $\mathrm{SF}_{6}$ as insulation in MV switchgear [3], [4]. Meeting clearance requirements in air-insulated medium voltage (MV) substations requires accurate withstand voltage prediction models of the dielectric design. Current models often fail to correctly predict the withstand voltage of such hybrid insulation systems. To understand how dielectric surfaces influence the breakdown voltage of an air-insulated gap, the breakdown mechanisms of the gap must be understood. The focus in this work is on positive lightning impulse (LI) stressed short (20-100 $\mathrm{mm}$ ) inhomogeneous air gaps, applicable to MV switchgear insulation designs. Breakdown in these gaps can happen via a leader-type channel propagating around the space charge left by the initial streamer discharges [5]. The aim of this work is

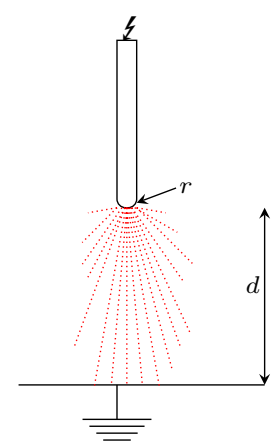

(a) Initial streamers cross- (b) Leader-type channel ing the gap

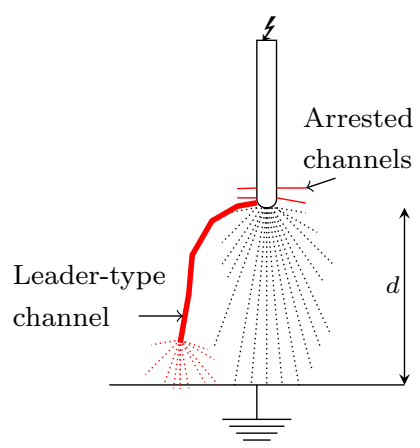

development around initial streamers
Fig. 1 - Illustration of discharge development by the leader-type breakdown mechanism in a rod-plane gap with clearance $d$ and a hemispheric rod of radius $r$.

to investigate how this breakdown mechanism fits with standard breakdown voltage prediction models.

\section{Breakdown in air}

\subsection{Empirical breakdown models}

Although primary streamers crossing the gap is sufficient to induce breakdown in weakly inhomogeneous fields, it is not a sufficient condition for breakdown in strongly inhomogeneous fields. An empirical model of streamer crossing is, however, traditionally used to predict the withstand voltage of strongly inhomogeneous gaps shorter than $1-2 \mathrm{~m}$ :

$$
U_{\mathrm{W}}=E_{\mathrm{st}} \cdot d+U_{0}
$$

where the statistical withstand voltage $U_{\mathrm{W}}$ is estimated based on an assumption of constant streamer channel field $E_{\mathrm{st}} \approx 0.54 \mathrm{kV} / \mathrm{mm}$ and a streamer head potential $U_{0}=20-30 \mathrm{kV}$ needed to cause breakdown [6]. The statistical withstand voltage $U_{\mathrm{W}}$ is defined from the $50 \%$ breakdown voltage as $U_{\mathrm{W}}=U_{50 \%}-3 \sigma$ [7].

Kojima et al. [5] classified positive LI breakdown mechanisms in rod-plane gaps. In their work, two main classes of positive breakdowns are described: channel-heating breakdown and leader-type breakdown. Channel-heating breakdown requires crossing and sufficient heating of a 
secondary streamer channel. These conditions can be met in gaps of a few $\mathrm{cm}$ if the voltage is high enough. Without a secondary streamer bridging the inter-electrode gap and heating the channel, breakdown will either not occur or it may be induced after the inception and propagation of a leader-type channel. If a leader-type channel develops, it typically starts at a different part of the rod than the primary streamer (Fig. 1) since there will be residual positive space charge from the streamer corona shielding the rod [8], [9].

\section{Method}

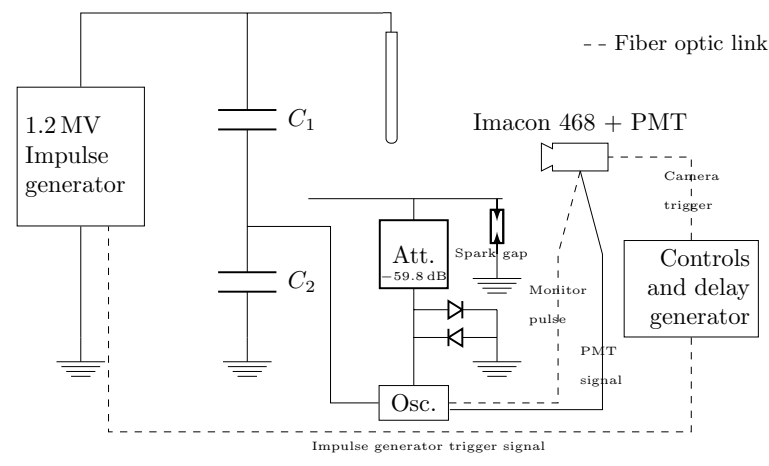

Fig. 2 - Experimental set-up for studying discharge behaviour in $20-100 \mathrm{~mm}$ rod-plane gaps. Impulse generator, camera, PMT, current measurement using attenuators (Att.) and current measurement protection (spark gaps and diodes) are shown.

\subsection{Camera and PMT}

A rod with a hemispheric tip of radius $r=3.5 \mathrm{~mm}$ or $10 \mathrm{~mm}$ was placed $20-100 \mathrm{~mm}$ over a $1 \times 1 \mathrm{~m}$ ground plane and stressed with $1.2 / 50 \mu$ s positive lightning impulses using a $1.2 \mathrm{MV}$ impulse generator. The experiments were performed in ambient air with the temperature, pressure and relative humidity being logged. An Imacon 468 ICCD camera with 7 frames of minimum exposure time $10 \mathrm{~ns}$ each was triggered with a delay generator to capture the spatiotemporal discharge development. An $85 \mathrm{~mm} \mathrm{f} / 1.8$ Nikkor lens was used with the camera which was placed inside a Faraday cage about $1 \mathrm{~m}$ away from the rod. A continuous signal of the light intensity was obtained using a PMT $2 \mathrm{~m}$ away. Two different PMTs, Philips 56UVP (160-650 nm) and Philips 56TVP (360-850 nm), were used with $2.5 \mathrm{kV}$ supply voltage and different light filters to study the emitted light of the discharges.

\subsection{Current measurement system}

The current was measured through a $23 \mathrm{~m} 50 \Omega$ signal cable (RG-214) with approximately $400 \mathrm{MHz}$ bandwidth connected to the ground plane. The signal cable was matched at the oscilloscope end after passing through a series of $13 \mathrm{GHz}$ T-type attenuators with a damping of up to $59.8 \mathrm{~dB}$. To protect the oscilloscope from breakdown currents, a $430 \mathrm{~V}$ spark gap was placed close to the ground plane, see Fig. 2. Two diodes were placed in anti-parallel close to the oscilloscope to arrest the fastest voltage transients. The spark gap voltage or attenuation can be modified to measure different current ranges, but a practical upper limit is given by the thermal rating of the first attenuator, $5000 \mathrm{~V}$ for $400 \mathrm{~ns}$.

The capacitance between the rod and plane, and between the plane and the supporting structure is in the $\mathrm{pF}$ range. With a $50 \mathrm{kV} 1.2 / 50 \mu$ s lightning impulse the peak of the capacitive charging current in a $10 \mathrm{pF}$ capacitor is around $I_{C}=0.5 \mathrm{~A}$. In addition to high frequency $(\mathrm{GHz})$ noise, a large damped oscillation with frequency $7.5-10 \mathrm{MHz}$ is induced in the current measurement system by the impulse generator as the setup is not placed in a Faraday cage.

\subsection{Digital post-processing}

The propagation times in the PMT, current and voltage measurement cables were found using a pulse generator. These cable delays and the internal PMT delay were compensated in the digital post-processing of the $5 \mathrm{GS} / \mathrm{s}$ oscilloscope recordings. The correct timing of the camera monitor pulse was found using a PMT and a fast lightemitting diode. A Python script that filters out parts of the current measurement noise was made. The script cuts the $7.5-10 \mathrm{MHz}$ frequencies in the frequency domain to remove noise and subtracts a similarly filtered current measurement without discharge activity in the time domain to remove capacitive current. The original current measurement is also plotted in the results.

As the discharges are faint, the image brightness and contrast were enhanced with photo-editing software. These parameters were adjusted to the same levels in all image series to normalise the evaluation of discharge intensity. Images of background light were subtracted to normalise intensities of the ICCDs.

\subsection{Breakdown voltages}

The $50 \%$ breakdown and inception voltages of the different rod-plane configurations were estimated using the up and down method with $n=20$ shots [10].

\section{Results}

\subsection{Breakdown voltage levels}

$50 \%$ breakdown $\left(U_{50 \%}\right)$ and inception $\left(U_{i, 50 \%}\right)$ voltages for the tested geometries are shown in Fig. 8. $50 \%$ breakdown voltages fit well with the empirical streamer propagation criterion in (1) using $U_{0}=20 \mathrm{kV}$. The $50 \%$ $\mathrm{BD}$ voltages for the strongly inhomogeneous fields seem to be independent of rod geometry, but a discrepancy is 

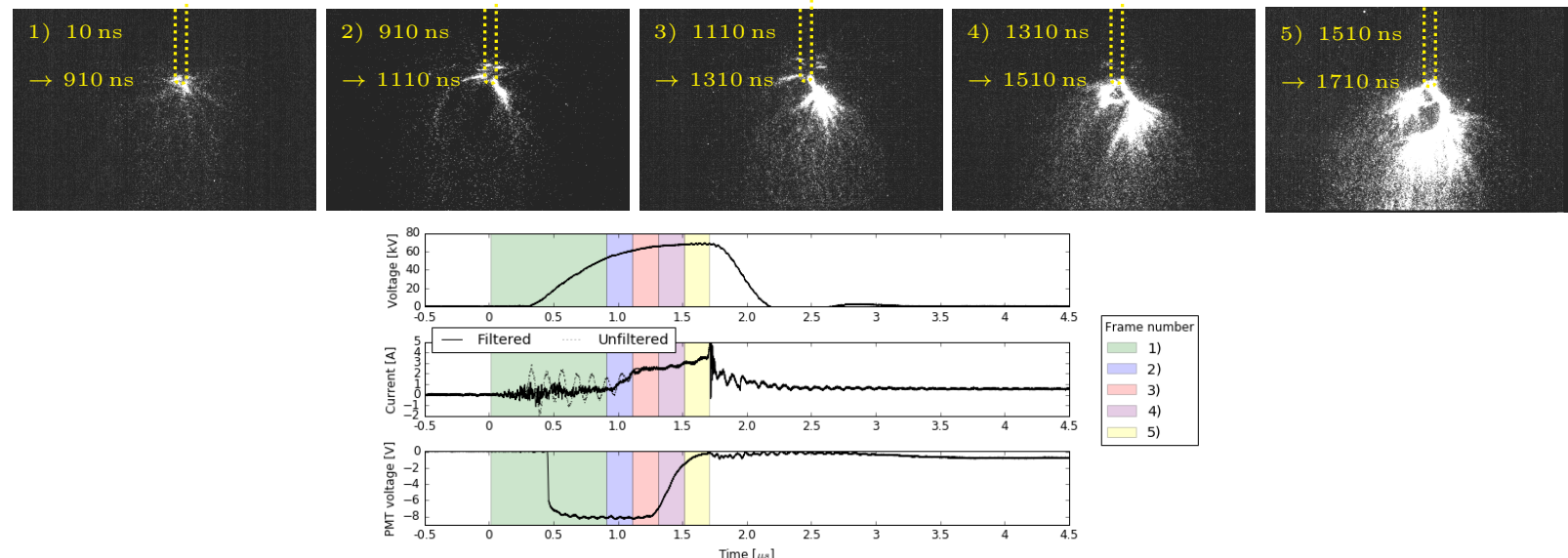

Fig. 3 - Breakdown development images and oscilloscope recordings, $d=60 \mathrm{~mm}, r=3.5 \mathrm{~mm}, U=69.66 \mathrm{kV}$. PMT wavelength detection range $160-650 \mathrm{~nm}$. Atmospheric pressure $1.009 \mathrm{bar}, 22^{\circ} \mathrm{C}$, relative humidity $36 \%$. Leader-type breakdown channel mechanims.
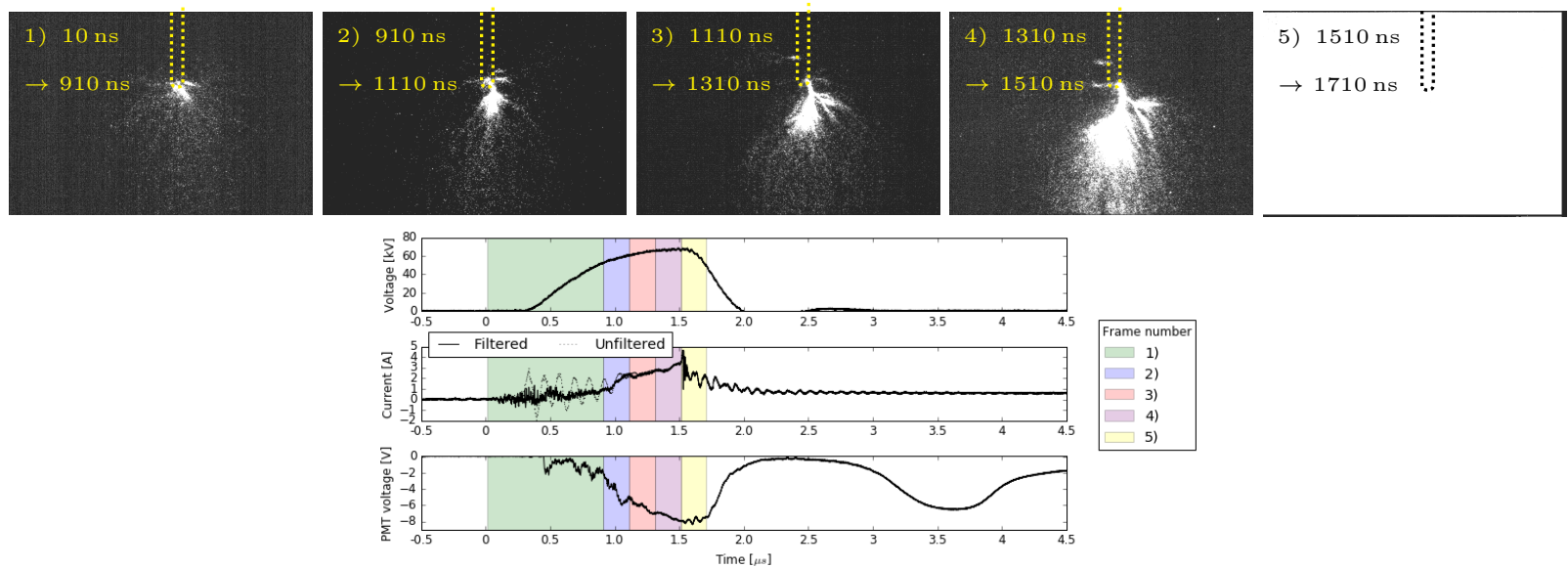

Fig. 4 - Breakdown development images and oscilloscope recordings, $d=60 \mathrm{~mm}, r=3.5 \mathrm{~mm}, U=68.75 \mathrm{kV}$. PMT wavelength detection range $610-850 \mathrm{~nm}$. Atmospheric pressure 1.009 bar, $22{ }^{\circ} \mathrm{C}$, relative humidity $36 \%$. Leader-type channel breakdown mechanism.

seen in the shorter gaps $(20-40 \mathrm{~mm})$.

\subsection{Discharge development}

High-speed images of leader-type breakdowns (Fig. 3 to 6) or channel-heating breakdown (Fig. 7) with corresponding voltage, current and PMT signal are shown. All image series have five or six frames of different timing and exposure time showing the discharge development. The positioning of the frames relative to oscilloscope recordings of voltage, current and light are indicated using color-shaded areas in the oscilloscope plots.

Breakdown occurs in all presented image series. Image series without breakdown usually showed the initial streamer activity only (frame 1 in Fig. 3 to 5 ) or the initial streamers and a leader-type channel stem that was stopped after propagating a few $\mathrm{mm}$ in a lateral direction.
These arrested channels were sometimes also seen when breakdown occurred as in Fig. 3 and 4 and as illustrated in Fig. 1b.

Two types of PMTs were combined with various filters so that different ranges of wavelengths were detected. Whereas Fig. 3 show the wavelength ranges 160-650 nm and Fig. 4 and 7 shows $610-850$ nm, Fig. 5 and 6 show the wavelengths $495-650 \mathrm{~nm}$.

In all image series, the discharge starts with a cloud of streamers crossing the gap as illustrated in Fig. 1a. This discharge activity was captured in a single long frame of $900 \mathrm{~ns}$ in all image series, so it is not possible to determine the propagation speed from the images. Previous work by the authors on similar gaps with dielectric barriers showed that these streamers move with roughly $2 \mathrm{~mm} / \mathrm{ns}$, crossing the gap in some tens of ns [4]. 

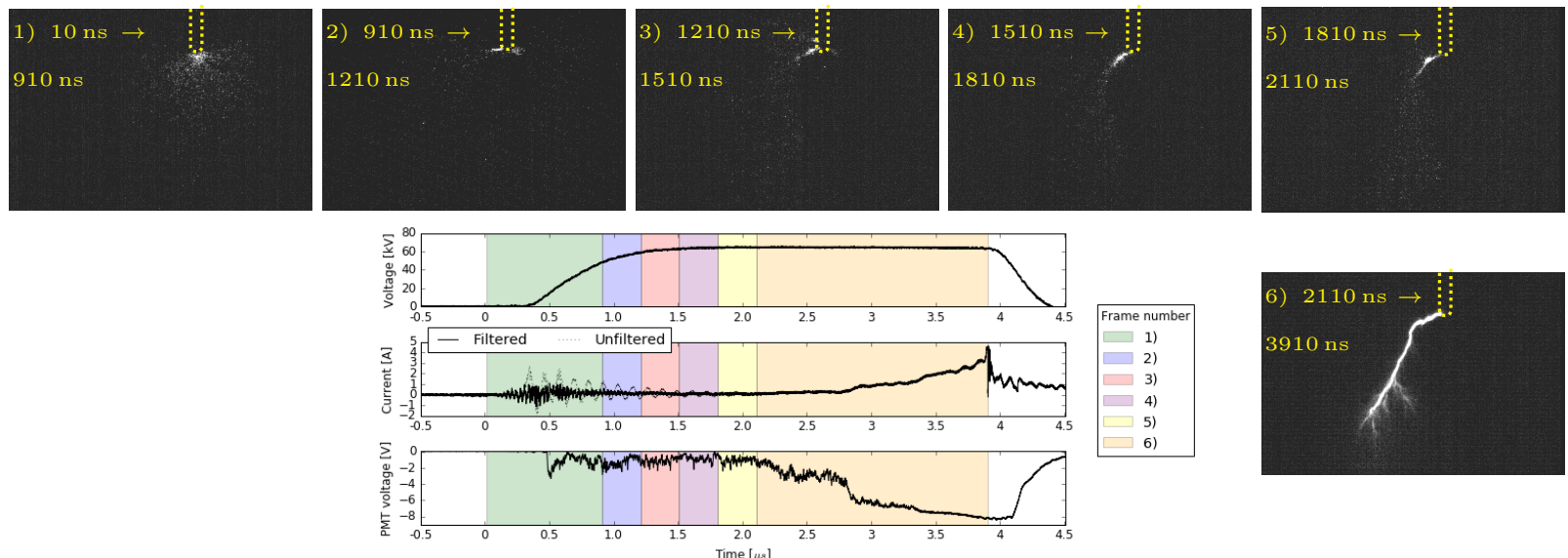

Fig. 5 - Breakdown development images and oscilloscope recordings, $d=80 \mathrm{~mm}, r=3.5 \mathrm{~mm}, U=65.71 \mathrm{kV}$. PMT wavelength detection range $495-650 \mathrm{~nm} .1 .020$ bar, $22{ }^{\circ} \mathrm{C}$, relative humidity $37 \%$. Leader-type channel breakdown mechanism.
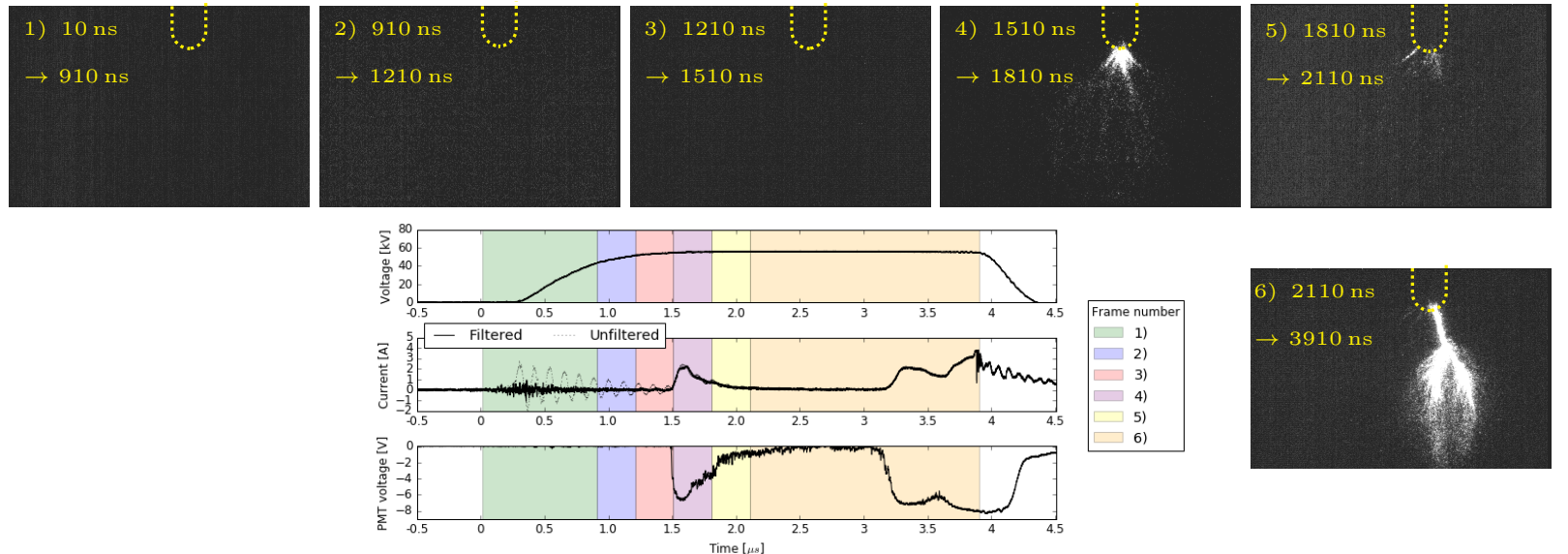

Fig. 6 - Breakdown development images and oscilloscope recordings, $d=60 \mathrm{~mm}, r=10 \mathrm{~mm}, U=56.58 \mathrm{kV}$. PMT wavelength detection range $495-650 \mathrm{~nm}$. Atmospheric pressure 1.005 bar, $22{ }^{\circ} \mathrm{C}$, relative humidity $36 \%$. Leader-type channel breakdown mechanism.

In the configurations where $d=20 \mathrm{~mm}$, leader-type channels were not observed. Instead, breakdown quickly developed after the initial streamer activity as can be seen in Fig. 7. The light in frames $1-4$ is probably from the secondary streamer activity and channel heating, while frame 5 and 6 show the breakdown channel.

For the $10 \mathrm{~mm}$ rod, the streamer current is typically around $2 \mathrm{~A}$, see purple shaded current area in Fig. 6 . The rise and fall times of the initial current are estimated from Fig. 6 to $50 \mathrm{~ns}$ and $500 \mathrm{~ns}$ respectively. The integrated charge is around $550 \mathrm{nC}$. Due to the initial oscillations, the initial streamer current is not visible in the originial current plots in Fig. 3 and 4. From the filtered current measurement it can be seen that the current is typically below $0.5 \mathrm{~A}$. The time from primary streamer inception to breakdown is variable and characterized by a dark period with lower light emission and current before the initiation of the leader-like channel.
After the primary streamer activity, the remaining frames in all figures show an illumination of a single leader-like channel propagating from the rod. The channel starts from different parts of the rod and seems to have a more or less pronounced lateral component in directly after initiation. The lateral propagation is easiest to see when the channel is propagating transverse to the camera axis as in Fig. 5. Several streamers connect the leader-type channel front to the ground plane as illustrated in Fig. $1 \mathrm{~b}$. Sometimes multiple or branched channels are observed as seen in Fig. 3 and 4. From frame $3-4$ in Fig. 4 it is estimated that the luminous channel moves with a speed of $0.1 \mathrm{~mm} / \mathrm{ns}$.

Whereas the initial streamer current seems to be relatively similar for the different geometries, the current induced in the plane during the leader-type channel propagation is varying.

Fig. 3 and 4 indicate that all parts of the discharge emit 

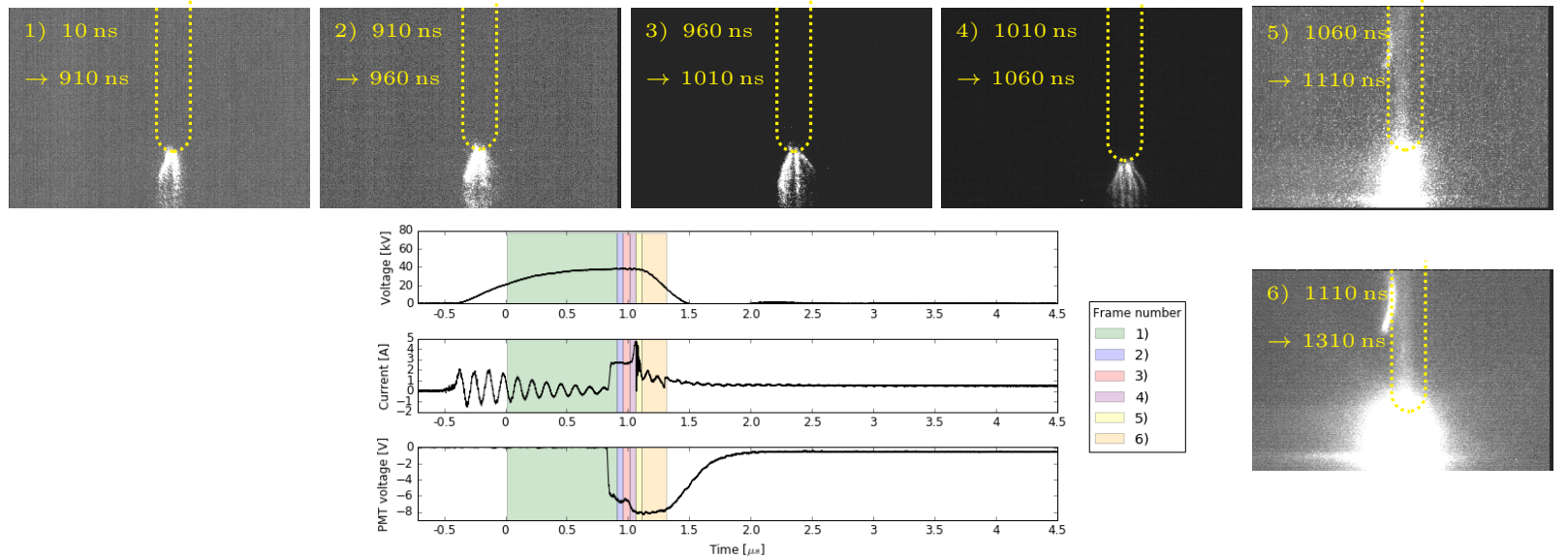

Fig. 7 - Breakdown development images and oscilloscope recordings, $d=20 \mathrm{~mm}, r=10 \mathrm{~mm}, U=38.76 \mathrm{kV}$. PMT wavelength detection range $610-850 \mathrm{~nm}$. Atmospheric pressure 0.999 bar, $22{ }^{\circ} \mathrm{C}$, relative humidity $35 \%$. Secondary streamer/channel-heating breakdown mechanism.

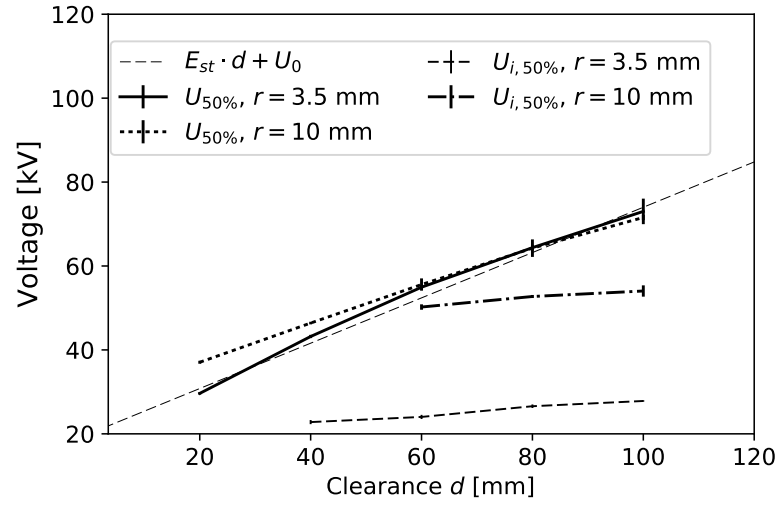

Fig. 8 - $50 \%$ breakdown $U_{50} \%$ voltages with $95 \%$ confidence intervals as a function of rod-plane separation $d$, compared with (1) using $U_{0}=20 \mathrm{kV}$

light in the wavelength range UV to IR. It was therefore not possible to clearly distinguish different discharge processes based on emitted light ranges only. However, the PMT in Fig. 3 goes directly into saturation with the initial streamers, which is not the case for Fig. 4 and 5. This indicates that the initial streamers emit light mostly in the part of the spectrum below $495 \mathrm{~nm}$, possibly down to UV.

\section{Discussion}

\subsection{Discharge development}

The period between primary streamers crossing and leader-type channel inception is sometimes characterized by propagation and crossing of secondary streamers [5], [11]. As the leader-type breakdown mechanism was the only breakdown mechanism seen when $d>20 \mathrm{~mm}$, it is assumed that a voltage higher than the $50 \% \mathrm{BD}$ voltage is needed in these geometries for secondary streamers to cause breakdown [5].
The initial lateral propagation of the leader-type channel is likely a result of the field distortion from the primary streamer discharges. The variability of the current induced by the leader-type channel could be a result of the stochastic nature of the channel propagation and branching.

The primary streamer current and charge depends on the inception level. The current will therefore be larger in geometries with greater homogeneity as inception levels are higher. As primary streamer discharges seem to emit light mostly at wavelengths below $495 \mathrm{~nm}$, it is assumed that the PMT signal in Fig. 4 during the leader-type channel propagation mainly comes from heating of the channel by the current supplying streamers in front of the channel.

\subsection{Breakdown voltage prediction models}

As breakdown is not induced by the initial streamers directly, it is possible that (1) should be interpreted as a leader-type channel propagation criterion instead of a streamer propagation criterion. Then the equation would represent the potential needed to launch a leader-type channel across the gap. Once the channel has propagated a few $\mathrm{cm}$ into the gap, it is likely to bridge the gap as streamers propagating from its head to ground increase the conductivity of the channel.

The breakdown voltage dependency on the size of the rod for gaps smaller than $40 \mathrm{~mm}$ (see Fig. 8) could be explained by the fact that the channel-heating breakdown mechanism evolves directly from the primary streamer discharges. As the inception voltage for primary streamer discharges increases with homogeneity, so does the breakdown voltage. The leader-type channel mechanism seen in gaps from $40-100 \mathrm{~mm}$ is on the other hand less dependent on rod geometry. A possible 
explanation is that this breakdown mechanism is less related to the initial discharges. This view is supported by the fact that it does not seem to evolve directly from the initial discharges, but rather propagates around them.

\section{Conclusions}

Breakdown mechanisms in inhomogeneous rod-plane gaps have been studied with a high-speed camera, PMTs and a current measurement system. The breakdown condition for larger gaps $(40-100 \mathrm{~mm})$ is inception of a leader-like channel after the crossing of a cloud of primary streamers. The channel is preceded by primary streamers incepting from the rod tip and propagating to ground. The leader-like channel does not follow the shortest path to ground as it is affected by the electric field distortion due to the space charges created by initial streamers. Similarly to leaders in long $(>1 \mathrm{~m})$ gaps, streamers are propagating from the leader-type channel head to ground.

The breakdown mechanism of the shortest gaps $(20 \mathrm{~mm})$ is governed by heating of initial streamer channels. This breakdown mechanism is dependent on the rod geometry as it evolves directly from the streamer channels. The empirical streamer propagation criterion (1) generally fits well with the $50 \%$ breakdown voltages for strongly inhomogeneous fields, and the predicted voltage of (1) could represent the potential needed for a leader-type channel to be initiated and propagate across the gap.

\section{Acknowledgement}

This work is part of the project "Electrical insulation with low-GWP gases" (project number: 245422) funded by the Research Council of Norway and the industrial partners ABB AS, Norway and ABB Switzerland Ltd.. The authors would also like to thank Dag Linhjell at SINTEF Energy Research, Norway, for all his help with the experimental set-up.

\section{References}

[1] J. Mantilla, N. Gariboldi, S. Grob, and M. Claessens, "Investigation of the insulation performance of a new gas mixture with extremely low GWP," in Electrical Insulation Conference (EIC), 2014, Jun. 2014, pp. 469-473.

[2] Y. Kieffel and F. Biquez, "SF6 alternative development for high voltage switchgears," in 2015 IEEE Electrical Insulation Conference (EIC), Jun. 2015, pp. 379-383.

[3] F. Mauseth, J. S. Jørstad, and A. Pedersen, "Streamer inception and propagation for air insulated rod-plane gaps with barriers," in 2012 Annual Report Conference on Electrical Insulation and Dielectric Phenomena (CEIDP), Oct. 2012, pp. 739-732.
[4] H. K. Meyer, F. Mauseth, A. Pedersen, and J. Ekeberg, "Streamer propagation in rod-plane air gaps with a dielectric barrier," in 2016 IEEE Conference on Electrical Insulation and Dielectric Phenomena (CEIDP), Oct. 2016, pp. 1037-1040.

[5] H. Kojima, K. Hotta, T. Kitamura, N. Hayakawa, A. Otake, K. Kobayashi, T. Kato, T. Rokunohe, and H. Okubo, "Classification of impulse breakdown mechanisms under non-uniform electric field in air," IEEE Transactions on Dielectrics and Electrical Insulation, vol. 23, no. 1, pp. 194-201, Feb. 2016.

[6] A. Pedersen, T. Christen, A. Blaszczyk, and H. Böhme, "Streamer inception and propagation models for designing air insulated power devices," in IEEE Conference on Electrical Insulation and Dielectric Phenomena, 2009. CEIDP '09, Oct. 2009, pp. 604-607.

[7] J. Kuffel and P. Kuffel, High Voltage Engineering Fundamentals. Newnes, Jul. 17, 2000, 556 pp.

[8] T. Kitamura, H. Kojima, N. Hayakawa, K. Kobayashi, T. Kato, and T. Rokunohe, "Influence of space charge by primary and secondary streamers on breakdown mechanism under non-uniform electric field in air," in 2014 IEEE Conference on Electrical Insulation and Dielectric Phenomena (CEIDP), Oct. 2014, pp. 122-125.

[9] I. Gallimberti, "The mechanism of the long spark formation," in Journal De Physique, Colloquium C, vol. 7, 1979, pp. 193-250.

[10] W. J. Dixon and A. M. Mood, "A method for obtaining and analyzing sensitivity data," Journal of the American Statistical Association, vol. 43, no. 241, pp. 109-126, 1948.

[11] R. S. Sigmond, "The residual streamer channel: Return strokes and secondary streamers," Journal of Applied Physics, vol. 56, no. 5, pp. 1355-1370, Sep. 1, 1984. 